Published in "Mathematische Zeitschrift 257(1): 7-12, 2007"

which should be cited to refer to this work.

\title{
Nonnegative curvature and cobordism type
}

\author{
Anand Dessai · Wilderich Tuschmann
}

\begin{abstract}
We show that in each dimension $n=4 k, k \geq 2$, there exist infinite sequences of closed simply connected Riemannian $n$-manifolds with nonnegative sectional curvature and mutually distinct oriented cobordism type.
\end{abstract}

Keywords Nonnegative curvature · Cobordism type · Finiteness theorems

Mathematics Subject Classification (2000) Primary 53C20

\section{Introduction}

This article is motivated by the finiteness theorems in Riemannian geometry (see, e.g., $[1,4-6,8,10,11])$ and the following

Fact 1.1 For any fixed $n \in \mathbb{N}, D>0$ and $c, C \in \mathbb{R}$ there are only finitely many oriented cobordism classes of closed smooth oriented $n$-manifolds which admit Riemannian metrics with sectional curvature $c \leq \sec \leq C$ and diameter $\leq D$.

Indeed, recall that closed oriented manifolds are oriented-cobordant if and only if they have the same Pontrjagin and Stiefel-Whitney numbers [13]. To establish Fact 1.1, it thus suffices to know that a both-sided curvature and upper diameter bound restrict the Pontrjagin numbers to finitely many possibilities. This, however, is

W. Tuschmann's research was supported in part by a DFG Heisenberg Fellowship.

\footnotetext{
A. Dessai

Department of Mathematics, University of Fribourg, Chemin du Musee 23, 1700 Fribourg, Switzerland

e-mail: anand.dessai@unifr.ch

W. Tuschmann $(\bowtie)$

Department of Mathematics, University of Kiel, Ludewig-Meyn-Strasse 4, 24098 Kiel, Germany

e-mail: tusch@math.uni-kiel.de
} 
a direct consequence of Chern-Weil theory, since those bounds also yield an upper bound for the volume.

The main result of this note shows that oriented cobordism finiteness does not continue to hold if besides the upper diameter one only assumes a lower curvature bound. This is not even the case for manifolds of nonnegative sectional curvature (where one may normalize the diameter to one). Using the work of Borel and Hirzebruch on homogeneous bundles [2,3] and the recent work of Grove and Ziller [7] we show

Theorem 1.2 In every dimension $n=4 k, k \geq 2$, there exist infinite sequences of closed simply connected Riemannian $n$-manifolds with nonnegative sectional curvature and mutually distinct Pontrjagin numbers. In particular, in all these dimensions there are infinitely many oriented cobordism types of nonnegatively curved manifolds.

Notice that $n=8$ is indeed the lowest dimension where this can happen, since Gromov's Betti number theorem and the fact that the first Pontrjagin number of a closed oriented four-manifold $M$ is proportional to its signature imply that a lower curvature and upper diameter bound restrict the oriented cobordism type of $M$ always to finitely many possibilities.

Theorem 1.2 also complements the following related infiniteness results in nonnegative sectional curvature (none of which, however, yield an infinite number of oriented cobordism types of closed nonnegatively curved $n$-manifolds):

- In each dimension $n \geq 6$ there exist infinite sequences of closed simply connected nonnegatively curved $n$-manifolds of mutually non-isomorphic integral homology ([7]; use Corollaries 3.7 and 3.9 and cross with simply connected nonnegatively curved Riemannian manifolds of appropriate codimension).

- In all dimensions $n \geq 9$ there exist infinite sequences of closed nonnegatively pinched Riemannian $n$-manifolds with uniformly bounded diameter and pairwise non-isomorphic rational cohomology rings ([12]; use Theorem 3.1 and cross with nonnegatively curved Riemannian manifolds of appropriate codimension).

- In each dimension $n \geq 10$ there exist infinite sequences of homotopy equivalent but mutually non-homeomorphic closed simply connected Riemannian $n$-manifolds with $0 \leq \sec \leq 1$, positive Ricci curvature and uniformly bounded diameter ([9], Theorem A).

To conclude the introduction, we give a short description of the proof of Theorem 1.2. In the following section we use Borel-Hirzebruch theory to exhibit an infinite sequence of $\mathbb{C P}^{2}$-bundles over $S^{4}$ with structure group $S O(3)$ whose total spaces have pairwise distinct oriented cobordism type. By results of Grove and Ziller all these total spaces admit Riemannian metrics of nonnegative sectional curvature. Crossing these examples with complex projective spaces of appropriate codimension then establishes Theorem 1.2 in all dimensions.

\section{$2 \mathbb{C P}^{2}$-bundles over $S^{4}$ and the proof of Theorem 1.2}

In this section we will first investigate the oriented cobordism type of the total space of certain $\mathbb{C P}^{2}$-bundles over $S^{4}$ (see Proposition 2.2) and then prove Theorem 1.2. We shall use the work of Borel and Hirzebruch on homogeneous bundles and refer to $[2,3]$ for details. Throughout what follows we will employ cohomology with integer coefficients, but rational coefficients would work as well. 
Let $\xi$ be a principal $S O(3)$-bundle over $S^{4}$ and let $E \rightarrow S^{4}$ be the associated three-dimensional real vector bundle. To $E$ we associate a $\mathbb{C P}^{2}$-bundle defined by taking the projectification of the complexified bundle $E \otimes \mathbb{C}$. Any such $\mathbb{C P}^{2}$-bundle can be obtained as a pullback of the following universal construction, which will be important for later computations.

Let $E G \rightarrow B G$ be a universal $G$-principal bundle for $G:=U(3)$ and let $H:=$ $U(2) \times U(1) \subset U(3)$. When applied to the universal $G$-principal bundle and to the $G$-space $G / H \cong \mathbb{C P}^{2}$, the Borel construction yields a bundle $\eta$ with total space $E(\eta):=E G \times{ }_{G} G / H$, base space $B(\eta):=B G$, fibre $G / H$ and projection $\pi: E(\eta) \rightarrow$ $B(\eta)$. Notice that $E(\eta)$ can be identified with the classifying space $B H:=E G / H$ for $H$-principal bundles via the map $E(\eta) \rightarrow E G / H,(e, g H) \sim \mapsto e g H$. We shall identify $B H$ with $E(\eta)$ and shall also write $\pi: B H \rightarrow B G$ for the projection $\pi: E(\eta) \rightarrow B(\eta)$.

The bundle $\eta$ is a universal $\mathbb{C P}^{2}$-bundle with structure group $G$. In particular, $\eta$ pulls back to the $\mathbb{C P}^{2}$-bundle associated to $\xi$. More precisely, let $f_{\xi}: S^{4} \rightarrow B G$ be the composition of a classifying map $S^{4} \rightarrow B S O(3)$ for $\xi$ and the map $B S O(3) \rightarrow B G$ induced from complexification. Then $\eta_{\xi}:=f_{\xi}^{*}(\eta)$ is, up to isomorphism, the $\mathbb{C} P^{2}$ bundle associated to $\xi$, and one has the following pullback diagram:

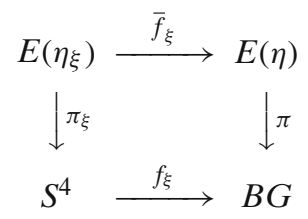

The total space $E\left(\eta_{\xi}\right)$ of $\eta_{\xi}$ is a closed connected eight-dimensional manifold which we will also denote by $M_{\xi}$. We fix an orientation of $S^{4}$ and fix the orientation of $M_{\xi}$ which is compatible with the one of $S^{4}$ and the orientation of the fibre $\mathbb{C P}^{2}$ (on $\mathbb{C P}^{2}$ we choose the orientation induced by the complex structure).

Let $\hat{\eta}_{\xi}$ denote the bundle along the fibres of $\eta_{\xi}$ and let $\hat{\eta}$ be the bundle along the fibres of $\eta$. Recall that $\hat{\eta}$ is a bundle over $B(\hat{\eta})=E(\eta)$ with total space $E(\hat{\eta})=$ $E G \times_{G} T(G / H)$ (see [2, Sect. 7] ). By naturality, $\hat{\eta}_{\xi}$ is isomorphic to $\bar{f}_{\xi}^{*}(\hat{\eta})$.

The tangent bundle $T M_{\xi}$ of $M_{\xi}$ decomposes as the direct sum of the tangent bundle of $S^{4}$ (which is stably trivial) pulled back via $\pi_{\xi}$ and $\hat{\eta}_{\xi}$. Hence, the total Pontrjagin class of $M_{\xi}$ satisfies

$$
p\left(M_{\xi}\right)=p\left(T M_{\xi}\right)=p\left(\pi_{\xi}^{*}\left(T S^{4}\right)\right) \cdot p\left(\hat{\eta}_{\xi}\right)=p\left(\hat{\eta}_{\xi}\right)=p\left(\bar{f}_{\xi}^{*}(\hat{\eta})\right)=\bar{f}_{\xi}^{*}(p(\hat{\eta})) .
$$

We shall now describe the oriented cobordism type of $M_{\xi}$ in terms of the Pontrjagin class of $E$ (see Proposition 2.2 below). First we recall from [13] that the oriented cobordism group $\Omega_{8}^{S O}$ is isomorphic to $\mathbb{Z} \oplus \mathbb{Z}$ and that the cobordism type of an oriented closed eight-dimensional manifold $M$ is uniquely determined by the Pontrjagin numbers $\int_{M} p_{1}(M)^{2}$ and $\int_{M} p_{2}(M)$.

Since the signature of $M_{\xi}$ vanishes by its multiplicativity property, the Pontrjagin numbers of $M_{\xi}$ determine each other. Hence, the oriented cobordism type of $M_{\xi}$ is uniquely determined by its Pontrjagin number $\int_{M_{\xi}} p_{1}\left(M_{\xi}\right)^{2}$.

In the following it will be convenient to think of the evaluation map $\int_{M_{\xi}}$ : $H^{8}\left(M_{\xi}\right) \rightarrow \mathbb{Z}$ as the pushforward $\left(\pi^{M_{\xi}}\right) !: H^{*}\left(M_{\xi}\right) \rightarrow H^{*-8}(p t)$ restricted to degree 8 , where $\pi^{M_{\xi}}$ is the map which maps $M_{\xi}$ to a point pt. Let $\pi_{!}: H^{*}(B H) \rightarrow H^{*-4}(B G)$ denote the pushforward for $\pi: B H \rightarrow B G$ and $\left(\pi_{\xi}\right)$ ! the one for $\pi_{\xi}: M_{\xi} \rightarrow S^{4}$. 


\section{Lemma 2.1}

$$
\int_{M_{\xi}} p_{1}\left(M_{\xi}\right)^{2}=\int_{S^{4}} f_{\xi}^{*}\left(\pi_{!}\left(p_{1}(\hat{\eta})^{2}\right)\right) .
$$

Proof Using the factorization $\left(\pi^{M_{\xi}}\right) !=\left(\pi^{S^{4}}\right) ! \circ\left(\pi_{\xi}\right)$, naturality and (1), we obtain

$$
\begin{aligned}
\int_{M_{\xi}} p_{1}\left(M_{\xi}\right)^{2} & =\left(\pi^{M_{\xi}}\right) !\left(p_{1}\left(M_{\xi}\right)^{2}\right)=\left(\pi^{M_{\xi}}\right) !\left(p_{1}\left(\hat{\eta}_{\xi}\right)^{2}\right)=\left(\pi^{M_{\xi}}\right) !\left(\bar{f}_{\xi}^{*}\left(p_{1}(\hat{\eta})^{2}\right)\right) \\
& =\left(\pi^{S^{4}}\right) !\left(\left(\pi_{\xi}\right) !\left(\bar{f}_{\xi}^{*}\left(p_{1}(\hat{\eta})^{2}\right)\right)\right)=\left(\pi^{S^{4}}\right) !\left(f_{\xi}^{*}\left(\pi_{!}\left(p_{1}(\hat{\eta})^{2}\right)\right)\right) \\
& =\int_{S^{4}} f_{\xi}^{*}\left(\pi !\left(p_{1}(\hat{\eta})^{2}\right)\right) .
\end{aligned}
$$

Next we express the Pontrjagin number $\int_{M_{\xi}} p_{1}\left(M_{\xi}\right)^{2}$ in terms of the Pontrjagin class of the 3-dimensional vector bundle $E$ over $S^{4}$ associated to $\xi$.

\section{Proposition 2.2}

$$
\int_{M_{\xi}} p_{1}\left(M_{\xi}\right)^{2}=21 \int_{S^{4}} p_{1}(E) .
$$

Proof To prove the proposition we will first compute $\pi_{!}\left(p_{1}(\hat{\eta})^{2}\right)$ using the method of [2,3] und then apply Lemma 2.1. Let $T$ be the maximal torus of $H=U(2) \times U(1)$ consisting of diagonal matrices. Notice that $T$ is also a maximal torus of $G=U(3)$. Let $W(H)$ and $W(G)$ denote the Weyl groups of $H$ and $G$. In the following we will always identify the cohomology of $B H$ with the Weyl invariants $H^{*}(B T)^{W(H)}$ and identify the cohomology of $B G$ with $H^{*}(B T)^{W(G)}$. We recall from [3] that the pushforward $\pi_{!}: H^{*}(B H) \rightarrow H^{*-4}(B G)$ can be described in terms of the complementary roots of $H$ in $G$. More precisely, let $\pm \gamma_{i}$ denote the complementary roots of $H$, where we choose the sign such that the product $\prod_{i} \gamma_{i}$ is compatible with the orientation of the fibre $\mathbb{C P}^{2}$. Then the pushforward $\pi_{!}: H^{*}(B H) \rightarrow H^{*-4}(B G)$ is given by (cf. [3, Sect. 22.9])

$$
\pi_{!}(x)=\sum_{\sigma \in W(G) / W(H)} \sigma\left(\frac{x}{\prod_{i} \gamma_{i}}\right) .
$$

In our situation the complementary roots are $\gamma_{1}=x_{3}-x_{1}$ and $\gamma_{2}=x_{3}-x_{2}$, where $x_{1}, x_{2}, x_{3} \in H^{2}(B T ; \mathbb{Z})$ is the basis of the lattice $H^{2}(B T ; \mathbb{Z})$ which corresponds to the standard basis of the integral lattice of $T$. The cosets of $W(H)$ in $W(G)$ are represented by non-trivial cyclic permutations of $x_{1}, x_{2}, x_{3}$.

Since $W(G)$ acts on $H^{*}(B T)$ by permuting $x_{1}, x_{2}, x_{3}$, the cohomology of $B G$ is a polynomial ring in the elementary symmetric functions in $x_{1}, x_{2}, x_{3}$. We denote, as usual, these functions by $c_{i}, 1 \leq i \leq 3$, so that $c_{i} \in H^{2 i}(B G ; \mathbb{Z})$ is the universal ith Chern class for $G$-principal bundles. Similarly, the cohomology of $B H$ is a polynomial ring in $x_{3}$ and the elementary symmetric functions in $x_{1}, x_{2}$. 
For the computation of $\pi !\left(p_{1}(\hat{\eta})^{2}\right)$ we collect the following values of $\pi$ ! which can be easily obtained from (2):

$$
\pi_{!}(1)=0, \quad \pi_{!}\left(x_{3}\right)=0, \quad \pi_{!}\left(x_{3}^{2}\right)=1, \quad \pi_{!}\left(x_{3}^{3}\right)=c_{1}, \quad \pi_{!}\left(x_{3}^{4}\right)=c_{1}^{2}-c_{2} .
$$

Notice here that the first two identities follow directly from dimensional reasons. The third identity is equivalent to the fact that the integral cohomology ring of $\mathbb{C P}^{2}$ is a truncated polynomial ring.

Using this information we shall now compute $\pi_{!}\left(p_{1}(\hat{\eta})^{2}\right)$. Recall from [2] that the total Pontrjagin class of the bundle $\hat{\eta}$ along the fibres is given by $p(\hat{\eta})=\prod_{i}\left(1+\gamma_{i}^{2}\right)$. Hence,

$$
p_{1}(\hat{\eta})=\left(x_{3}-x_{1}\right)^{2}+\left(x_{3}-x_{2}\right)^{2}=\left(c_{1}^{2}-2 c_{2}\right)-2 c_{1} \cdot x_{3}+3 x_{3}^{2}
$$

and

$p_{1}(\hat{\eta})^{2}=\left(c_{1}^{2}-2 c_{2}\right)^{2}-\left(4\left(c_{1}^{2}-2 c_{2}\right) \cdot c_{1}\right) x_{3}+\left(6\left(c_{1}^{2}-2 c_{2}\right)+4 c_{1}^{2}\right) x_{3}^{2}-12 c_{1} \cdot x_{3}^{3}+9 x_{3}^{4}$.

This gives

$$
\pi_{!}\left(p_{1}(\hat{\eta})^{2}\right)=\left(6\left(c_{1}^{2}-2 c_{2}\right)+4 c_{1}^{2}\right) \pi_{!}\left(x_{3}^{2}\right)-12 c_{1} \cdot \pi_{!}\left(x_{3}^{3}\right)+9 \pi_{!}\left(x_{3}^{4}\right)=7 c_{1}^{2}-21 c_{2},
$$

where we have used Eqs. (3) and the fact that $\pi$ ! is a module homomorphism.

An application of Lemma 2.1 then completes the proof that

$$
\int_{M_{\xi}} p_{1}\left(M_{\xi}\right)^{2}=\int_{S^{4}} f_{\xi}^{*}\left(\pi_{!}\left(p_{1}(\hat{\eta})^{2}\right)\right)=\int_{S^{4}} f_{\xi}^{*}\left(7 c_{1}^{2}-21 c_{2}\right)=21 \int_{S^{4}} p_{1}(E) .
$$

Proof of Theorem 1.2 We shall first prove the theorem in dimension eight. The eightdimensional examples will be given by the total spaces $M_{\xi}$ of $\mathbb{C} P^{2}$-bundles over $S^{4}$ considered before.

Recall that isomorphism classes of oriented three-dimensional real vector bundles over $S^{4}$ are classified by $\pi_{3}(S O(3)) \cong \mathbb{Z}$ and distinguished by their first Pontrjagin class. According to Proposition 2.2, the corresponding eight-dimensional manifolds $M_{\xi}$ have pairwise distinct oriented cobordism type, distinguished by $\int_{M_{\xi}} p_{1}\left(M_{\xi}\right)^{2}$. By the work of Grove and Ziller all principal $S O(3)$-bundles over $S^{4}$ are known to carry invariant metrics of nonnegative sectional curvature and, hence, the associated homogeneous bundles $M_{\xi}$ admit nonnegatively curved metrics as well (see [7], Theorems $\mathrm{E}$ and $\mathrm{F}$ ). This completes the proof of Theorem 1.2 in dimension eight.

To exhibit an infinite sequence of closed simply connected Riemannian $4 k$-manifolds with nonnegative sectional curvature and mutually distinct oriented cobordism type for each $k>2$, it now suffices to consider the Riemannian products of the manifolds $M_{\xi}$ with $\mathbb{C P}^{2 k-4}$. In fact, the rational oriented cobordism ring $\Omega_{*}^{S O} \otimes \mathbb{Q}$ is a polynomial ring in the $\mathbb{C} P^{2 l}$ and, hence, multiplication with $\mathbb{C P}^{2 k-4}$ is injective in $\Omega_{*}^{S O} \otimes \mathbb{Q}$. This observation completes the proof of Theorem 1.2.

Question 2.3 All manifolds $M_{\xi}$ as well as all higher dimensional examples have pairwise non-isomorphic cohomology rings. Does Theorem 1.2 still hold if one fixes, in addition, the cohomology ring or the homotopy type of the manifolds in question? 


\section{References}

1. Anderson, M.T., Cheeger, J.: Diffeomorphism finiteness for manifolds with Ricci curvature and $L^{n / 2}$-norm of curvature bounded. Geom. Funct. Anal. 1, 231-252 (1991)

2. Borel, A., Hirzebruch, F.: Homogeneous spaces I. Am. J. Math. 80, 458-538 (1958)

3. Borel, A., Hirzebruch, F.: Homogeneous spaces II. Am. J. Math. 81, 315-382 (1959)

4. Cheeger, J.: Finiteness theorems for Riemannian manifolds. Am. J. Math. 92, 61-74 (1970)

5. Fang, F., Rong, X.: The second twisted Betti number and the convergence of collapsing Riemannian manifolds. Invent. Math. 150, 61-109 (2002)

6. Gromov, M.: Curvature, diameter and Betti numbers. Comment. Math. Helv. 56, 179-195 (1981)

7. Grove, K., Ziller, W.: Curvature and symmetry of Milnor spheres. Ann. Math. 152, 331-367 (2000)

8. Grove, K., Petersen, P., Wu, J.-Y.: Geometric finiteness theorems via controlled topology. Invent. Math. 99, 205-213 (1991)

9. Kapovitch, V., Petrunin, A., Tuschmann, W.: Nonnegative pinching, moduli spaces and bundles with infinitely many souls. J. Diff. Geom. 71, 365-383 (2005)

10. Peters, S.: Cheeger's finiteness theorem for diffeomorphism classes of Riemannian manifolds. J. Reine Angew. Math. 349, 77-82 (1984)

11. Petrunin, A., Tuschmann, W.: Diffeomorphism finiteness, positive pinching, and second homotopy. Geom. Funct. Anal. 9, 736-774 (1999)

12. Totaro, B.: Curvature, diameter, and quotient manifolds. Math. Res. Lett. 10, 191-203 (2003)

13. Wall, C.T.C.: Determination of the cobordism ring. Ann. Math. 72, 292-311 (1960) 